ARTICLE

\title{
Structural basis of heterotetrameric assembly and disease mutations in the human cis-prenyltransferase complex
}

Michal Lisnyansky Bar-El (10 1, Pavla Vaňková2,3, Adva Yeheskel ${ }^{4}$, Luba Simhaev ${ }^{4}$, Hamutal Engel ${ }^{4}$, Petr Man², Yoni Haitin ${ }^{1,5,7 凶} \&$ Moshe Giladi ${ }^{1,6,7 凶}$

The human cis-prenyltransferase (hcis-PT) is an enzymatic complex essential for protein Nglycosylation. Synthesizing the precursor of the glycosyl carrier dolichol-phosphate, mutations in hcis-PT cause severe human diseases. Here, we reveal that hcis-PT exhibits a heterotetrameric assembly in solution, consisting of two catalytic dehydrodolichyl diphosphate synthase (DHDDS) and inactive Nogo-B receptor ( $\mathrm{NgBR}$ ) heterodimers. Importantly, the 2.3 $\AA$ crystal structure reveals that the tetramer assembles via the DHDDS C-termini as a dimerof-heterodimers. Moreover, the distal $\mathrm{C}$-terminus of $\mathrm{NgBR}$ transverses across the interface with DHDDS, directly participating in active-site formation and the functional coupling between the subunits. Finally, we explored the functional consequences of disease mutations clustered around the active-site, and in combination with molecular dynamics simulations, we propose a mechanism for hcis-PT dysfunction in retinitis pigmentosa. Together, our structure of the hcis-PT complex unveils the dolichol synthesis mechanism and its perturbation in disease.

\footnotetext{
${ }^{1}$ Department of Physiology and Pharmacology, Sackler Faculty of Medicine, Tel-Aviv University, Tel-Aviv 6997801, Israel. ${ }^{2}$ Institute of Microbiology of the Czech Academy of Sciences, Division BioCeV, Prumyslova 595, 25250 Vestec, Czech Republic. ${ }^{3}$ Department of Biochemistry, Faculty of Science, Charles University, Hlavova 2030/8, 12843 Prague 2, Czech Republic. ${ }^{4}$ Blavatnik Center for Drug Discovery, Tel Aviv University, Tel Aviv 6997801, Israel. ${ }^{5}$ Sagol School of Neuroscience, Tel Aviv University, Tel Aviv 6997801, Israel. ${ }^{6}$ Tel Aviv Sourasky Medical Center, Tel Aviv 6423906, Israel. ${ }^{7}$ These authors jointly supervised this work: Yoni Haitin, Moshe Giladi. ${ }^{凶}$ email: yhaitin@tauex.tau.ac.il; moshegil@post.tau.ac.il
} 
P renyltransferases are essential enzymes that synthesize isoprenoids, an enormous group of chemically diverse compounds participating in a myriad of cellular processes in all living cells ${ }^{1}$. With chain lengths varying from $\mathrm{C}_{10}$ (geranyl diphosphate) to $>\mathrm{C}_{10,000}$ (natural rubber), isoprenoids are synthesized by chain elongation of an allylic diphosphate primer via a variable number of condensation reactions with isopentenyl pyrophosphate (IPP, $\left.\mathrm{C}_{5}\right)^{2-4}$. Prenyltransferases are classified as cis-prenyltransferase or trans-prenyltransferase according to the double bonds they form during the condensation reaction ${ }^{3}$. Cisprenyltransferases are further classified according to their product chain length into short-chain $\left(\mathrm{C}_{15}\right)$, medium-chain $\left(\mathrm{C}_{50-55}\right)$, longchain $\left(\mathrm{C}_{70-120}\right)$, and rubber synthases ${ }^{1}$. Importantly, while shortand medium-chain cis-prenyltransferase complexes are homodimeric, long-chain cis-prenyltransferases and rubber synthases are formed by a heteromeric subunit assembly of unknown stoichiometry $1,4,5$. To date, only homodimeric enzymes were structurally characterized ${ }^{6-9}$. Therefore, our understanding of the mechanisms allowing long-chain isoprenoid formation by heteromeric enzymes remains limited.

The human cis-prenyltransferase (hcis-PT) complex catalyzes the formation of dehydrodolichyl diphosphate (DHDD, $\mathrm{C}_{85-100}$ ), a long-chain isoprenoid, by chain elongation of farnesyl diphosphate $\left(\mathrm{FPP}, \mathrm{C}_{15}\right.$ ) via multiple condensations with IPP (Fig. 1a) ${ }^{10}$. DHDD is the precursor for dolichol-phosphate, the lipidic glycosyl carrier crucial for N-linked protein glycosylation (Fig. 1a) ${ }^{11}$. Localized to the endoplasmic reticulum, hcis-PT is composed of two structurally and functionally distinct subunit types. These include the catalytically active DHDD synthase (DHDDS) and the quiescent Nogo-B receptor (NgBR) subunits ${ }^{10}$. Importantly, while DHDDS subunits are cytosolic, NgBR can be subdivided into an $\mathrm{N}$-terminal transmembrane domain and a C-terminal pseudo cis-prenyltransferase domain (Supplementary Fig. 1), which lacks detectable catalytic activity and directly interacts with DHDDS $^{10}$.

In line with the crucial significance of N-linked glycosylation for proper cellular function, mutations in both hcis-PT subunits were associated with human diseases. Specifically, DHDDS missense mutations were shown to result in phenotypes ranging from autosomal recessive retinitis pigmentosa $(\mathrm{arRP})^{12,13}$, through developmental epileptic encephalopathies ${ }^{14}$, to a case of fatal congenital disorder of glycosylation reported in a patient heterozygous for both a splice site and a nonsense mutation ${ }^{15}$. Moreover, a missense mutation in the conserved C-terminal RxG a

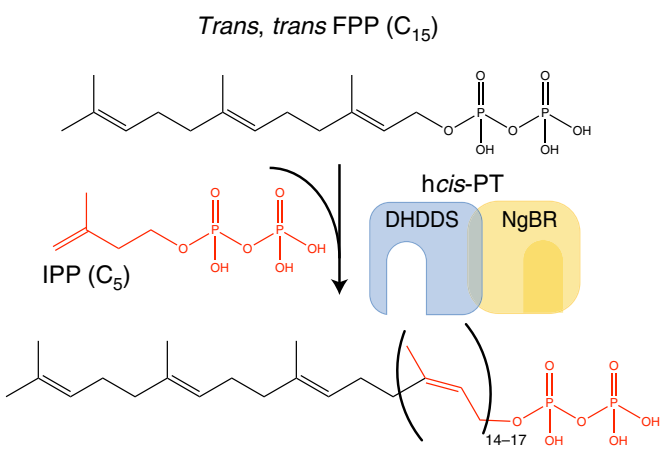

C

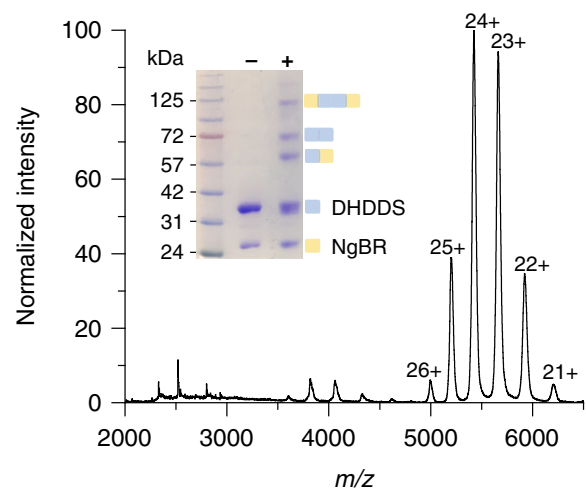

b

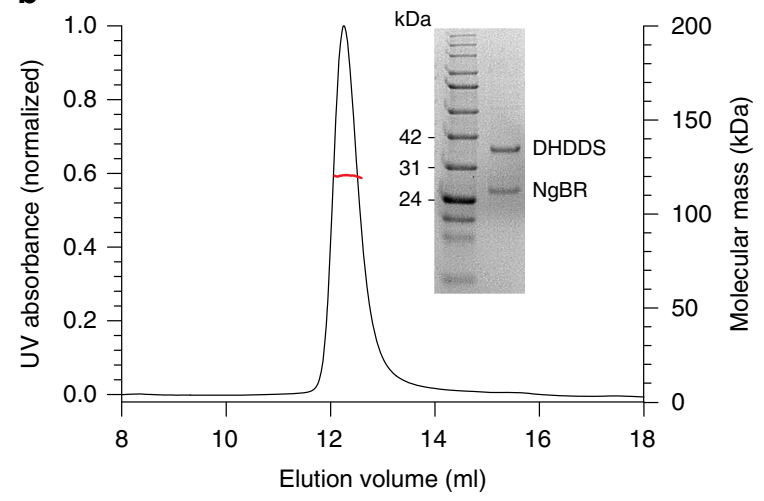

d

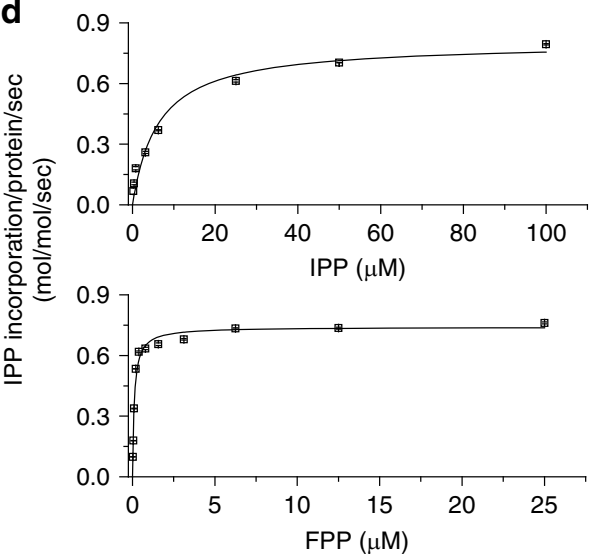

Fig. 1 Complex stoichiometry and functional analysis of shcis-PT. a Dehydrodolichyl diphosphate synthesis reaction scheme. The IPP moiety is colored red. The hcis-PT subunits DHDDS and NgBR are schematically drawn and colored blue and yellow, respectively. b Representative SEC-MALS analysis of the purified shcis-PT. The black and red curves indicate UV absorption and molecular mass, respectively. The experiment was performed twice using two different protein batches. Inset: SDS-PAGE analysis of the purified complex. Left lane: molecular weight marker, right lane: purified shcis-PT. Molecular weights $(\mathrm{kDa})$ are indicated. Source data are provided as a Source Data file. c Native ESI-MS spectrum obtained using low activation conditions. The main distribution corresponds to the heterotetramer (charge states 21-26). Inset: SDS-PAGE analysis of the complex following glutaraldehyde cross-linking. Left lane: molecular weight marker, middle lane: purified shcis-PT without glutaraldehyde, and right lane: purified shcis-PT with glutaraldehyde. Molecular weights $(\mathrm{kDa})$ are indicated. The experiment was performed twice using two different protein batches. The oligomeric state represented by each state is illustrated using the schematic presentation from panel (a). Source data are provided as a Source Data file. $\mathbf{d}$ In vitro activity of purified shcis-PT assessed as IPP incorporation. Experiments were performed as described in the "Methods" section. Data are presented as mean \pm SEM ( $n=3$ independent experiments). Source data are provided as a Source Data file. 
motif of $\mathrm{NgBR}$ was shown to cause a congenital glycosylation disorder with refractory epilepsy, visual and neurological impairments, congenital scoliosis, and hearing deficit ${ }^{16}$. Finally, recently identified missense mutations in NgBR were shown to contribute to the etiology of Parkinson's disease ${ }^{17}$. Intriguingly, the different pathogenic mutations in hcis-PT seem to have diverse effects on cellular glycosylation. For example, while the missense mutation in the RxG motif of NgBR led to reduced glycosylation in patients fibroblasts ${ }^{16}$, and the patient suffering from the fatal glycosylation disorder displayed hypoglycosylation of serum glycoproteins ${ }^{15}$, the arRP mutation in DHDDS does not seem to have any significant effect on glycosylation in a knock-in mouse model 18,19 , and some patients with DHDDS-related developmental epileptic encephalopathy display normal glycosylation assay results ${ }^{14}$. Thus, the interplay between the genotype and cellular phenotype may be more complex than originally thought and awaits further exploration.

We have previously shown that DHDDS can form functional homodimers, but these complexes exhibit poor catalytic activity compared to the homodimeric orthologs or the heteromeric hcis$\mathrm{PT}^{6,20,21}$. Accordingly, previous studies suggested that NgBR can allosterically modulate the activity of the catalytic DHDDS subunit ${ }^{10,16,21}$. Indeed, overexpression of NgBR was shown to significantly enhance hcis-PT activity in cells, supporting an NgBRmediated allosteric modulation of DHDDS activity ${ }^{10,16}$. This effect was suggested to involve a conserved RxG motif, localized to the NgBR C-terminal tail ${ }^{21}$. Recently, the structure of Nus1, the yeast homolog of $\mathrm{NgBR}$, was determined. The structure, devoid of the $\mathrm{N}$-terminal transmembrane domain, revealed that similarly to DHDDS, Nus1 can form homodimers when expressed alone ${ }^{22}$. However, Nus1 does not contain the canonical RxG motif. Instead, it contains a smaller and neutral asparagine residue $^{22}$. Thus, the quest for elucidating the functional and structural roles of NgBR activity in the context of the hcis-PT complex is still ongoing and the underlying mechanism remains to be determined.

Despite an immense body of work focusing on the biochemical and structural properties of cis-prenyltransferases, our mechanistic understanding of these enzymes arises mainly from investigations of homodimeric prokaryotic, plant, and fungal orthologs ${ }^{2,4}$. Thus, in contrast with the growing clinical relevance of hcis-PT, the basic mechanisms underlying its heteromeric assembly, intersubunit communication, and long-chain isoprenoid synthesis remain poorly understood. Recently, the structure of DHDDS in complex with NgBR devoid of its transmembrane region was determined in complex with IPP, and was reported to exhibit a heterodimeric assembly ${ }^{23}$. Here, we established a similar overexpression and co-purification paradigm of the hcis-PT complex devoid of its transmembrane region. Unexpectedly, we show that the purified complex formed stable heterotetramers in solution, with equimolar stoichiometry of DHDDS and NgBR subunits. Moreover, it exhibited a marked activity enhancement compared with the purified homodimeric DHDDS $^{20}$. Next, we determined the co-crystal structure of the complex with bound FPP, phosphate, and $\mathrm{Mg}^{2+}$ at $2.3 \AA$ resolution. While DHDDS encompasses an active-site reminiscent to that observed in homodimeric family members, the structure exposes the long-sought involvement of NgBR in active-site organization and provides insights into the molecular mechanisms associated with the functional enhancement it confers. Moreover, it reveals the structural organization of the DHDDS Cterminal domain, exhibiting a "helix-turn-helix" motif fold not observed in other cis-prenyltransferases, which facilitates complex tetramerization via a dimer-of-heterodimers assembly mode. Importantly, molecular dynamics (MD) simulations pinpoint the mechanisms leading to hcis-PT dysfunction in arRP. Finally, the structure lays the foundation toward identifying the determinants governing long-chain isoprenoid synthesis.

\section{Results}

Purification and in vitro activity characterization of the soluble hcis-PT complex. Previously, sequence and biochemical analyses of NgBR revealed that it interacts with DHDDS via its cytosolic C-terminal pseudo cis-prenyltransferase homology domain (Supplementary Fig. 1) ${ }^{10}$. Thus, we generated an NgBR construct solely encompassing its cytosolic domain (sNgBR, residues $73^{*}-293^{*}$, where the asterisks designate $\mathrm{NgBR}$ residues). Importantly, previous studies using yeast complementation showed that truncation up to position $85^{*}$ did not affect the ability of $\mathrm{NgBR}$ to support cell growth following co-transformation with DHDDS, indicating that the catalytic function of the complex is preserved in the absence of the transmembrane domain ${ }^{21}$. Next, we cooverexpressed the full-length human DHDDS (residues 1-333) and $\mathrm{sNgBR}$ in Escherichia coli. Following purification, we obtained a homogeneous population of heteromeric soluble hcisPT (shcis-PT) (Fig. 1b).

Intriguingly, during the final size-exclusion purification step, we noticed that the elution volume of shcis-PT corresponds to a higher-than-expected molecular weight range. Size-exclusion chromatography multiangle light-scattering (SEC-MALS) analysis of the purified shcis-PT revealed a monodispersed population with a molecular weight of $119.7 \pm 0.4 \mathrm{kDa}$ (Fig. 1b). Within the experimental error of SEC-MALS, this mass may correspond to a stable heterotetramer composed of either two DHDDS (monomer molecular weight $=39.1 \mathrm{kDa}$ ) and two sNgBR (monomer molecular weight $=25.2 \mathrm{kDa}$ ) subunits or one DHDDS and three sNgBR subunits. In order to determine the stoichiometry of the complex, we used native electrospray ionization (ESI) mass spectrometry (MS) (Fig. 1c). Importantly, native ESI spectra, obtained at low activation conditions to preserve the tertiary structure, revealed a mass of $130.1 \pm 0.2 \mathrm{kDa}$, confirming the heterotetrameric organization of the complex with a stoichiometry of two DHDDS and two sNgBR subunits (Fig. 1c). However, under these conditions, the deconvoluted mass was higher than predicted due to the presence of sodium cations and low-molecular-weight adducts. Indeed, along with partial complex disintegration upon stepwise activation, a shift of the heterotetrameric population toward lower mass $(128.7 \pm 0.02$ $\mathrm{kDa}$ ) was observed, and an agreement between theoretical and calculated masses was achieved (Supplementary Fig. 2). Finally, we used a cross-linking approach that enables the identification of subunit interactions within the heterotetramer. Treatment with glutaraldehyde, a homobifunctional amine-reactive cross-linker, resulted in the emergence of high-order oligomers, culminating in a heterotetrameric complex with a mass of $\sim 125 \mathrm{kDa}$ (Fig. 1c). In addition, although the cross-linking treatment can theoretically yield three types of dimers (DHDDS homodimer, sNgBR homodimer, and DHDDS-sNgBR heterodimer), we could clearly detect only two bands, corresponding to DHDDS homodimers and DHDDS-sNgBR heterodimers, suggesting that the $\mathrm{sNgBR}$ subunits exhibit spatial separation in the context of shcis-PT. Together, the biophysical and biochemical characterizations of shcis-PT suggest a dimer-of-heterodimers arrangement.

Next, in order to validate that the purified complex is catalytically viable, we tested its activity in vitro using a radioligand-based assay (Fig. 1d). The purified shcis-PT exhibited $k_{\text {cat }}=0.74 \pm 0.02 \mathrm{~s}^{-1}$ and $K_{\mathrm{M}}=6.23 \pm 1.50$ and $0.11 \pm 0.01 \mu \mathrm{M}$ for IPP and FPP, respectively. The $k_{\text {cat }}$ value is similar to that previously reported for the intact hcis-PT ${ }^{21}$ and $\sim 400$-fold higher compared to that of homodimeric DHDDS ${ }^{20}$. These results demonstrate that shcis-PT recapitulates the function of the intact 
a
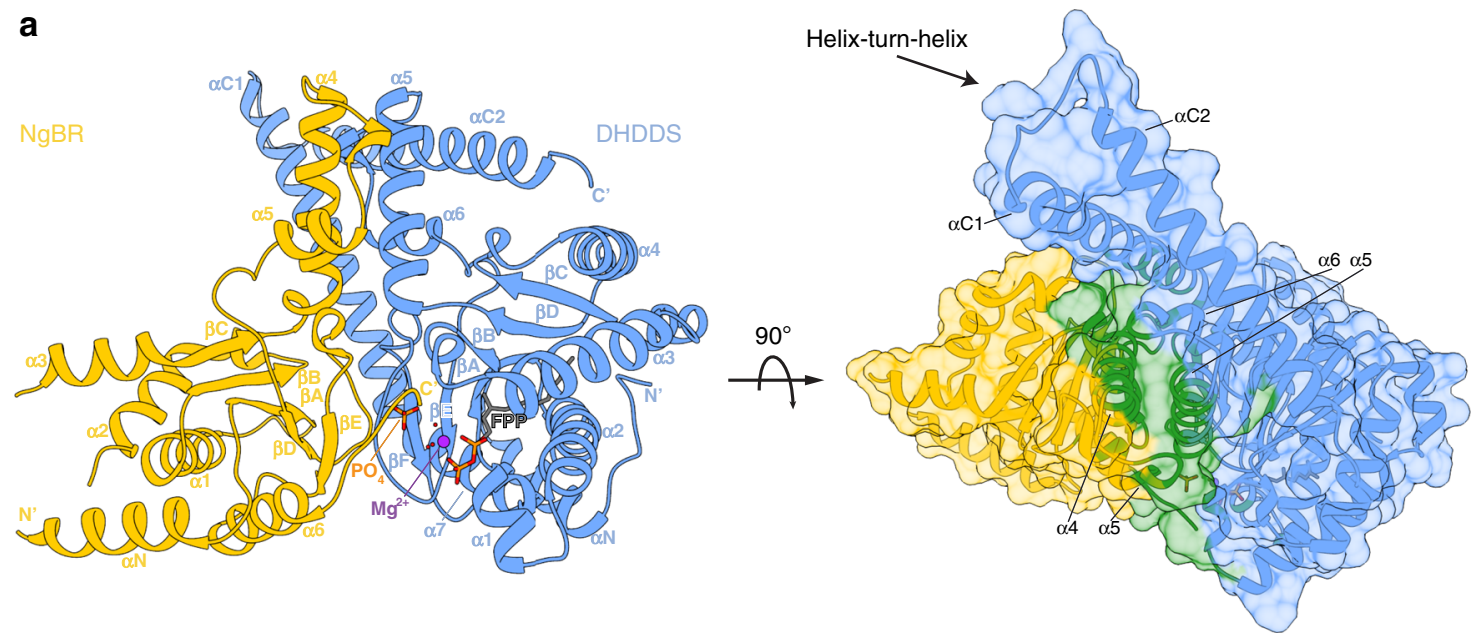

b

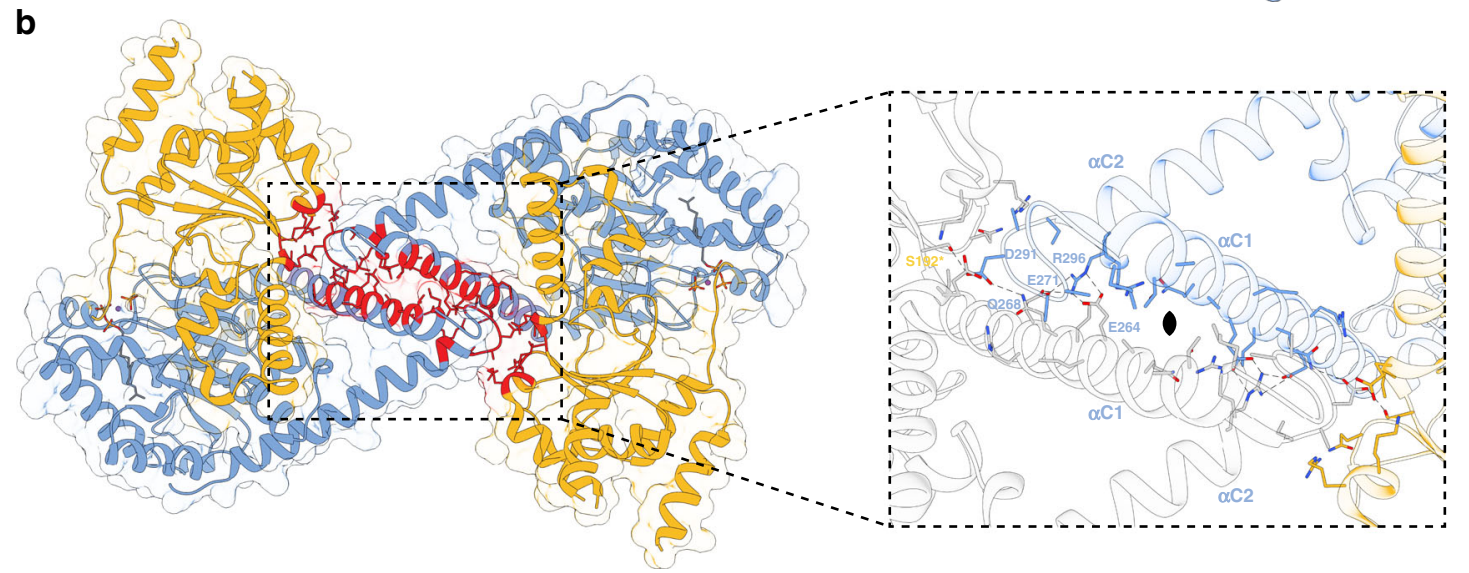

Fig. 2 Overall structure of xcis-PT. a Left: cartoon representation of the ASU, composed of a DHDDS-sNgBR $167^{\star}-175^{\star}$ heterodimer. DHDDS is colored blue and NgBR is colored yellow. Secondary structure elements are labeled. Right: surface representation of the ASU with residues involved in the heterodimeric interface highlighted in green. $\mathbf{b}$ The heterotetrameric complex assembly mode. Residues involved in tetramerization are shown as sticks and colored red. The rectangle frames a zoom perspective of the interactions between one heterodimer (colored as in panel A) and a heterodimer from the neighboring ASU (colored white).

complex, further reinforcing that the absence of the N-terminus of $\mathrm{NgBR}$ does not impair the catalytic activity of the complex. Moreover, the increased activity of the heteromeric complex compared with homodimeric DHDDS points toward an intersubunit communication mode, since NgBR lacks catalytic activity $^{1}$.

Structure overview of the shcis-PT subunits. In order to unveil the structural basis of the functional coupling observed in the context of the shcis-PT, we sought to determine its structure using $\mathrm{X}$-ray crystallography. However, as initial crystallization attempts were unsuccessful, we removed residues $167^{*}-175^{*}$ from $\mathrm{NgBR}$ $\left(\operatorname{sNgBR} \Delta 167^{*}-175^{*}\right)$, corresponding to an unresolved loop in the structure of the yeast homolog Nus1 ${ }^{22}$, assuming that this region is highly flexible and precludes crystallization. Indeed, this construct (termed hereafter xcis-PT) resulted in well-diffracting crystals while demonstrating unperturbed catalytic activity (Supplementary Fig. 3), allowing us to determine its structure in complex with $\mathrm{Mg}^{2+}$ and FPP at $2.3 \AA$ resolution (Supplementary Fig. 4). The asymmetric unit (ASU) contains a single heterodimer (Fig. 2a), with $\mathrm{Mg}^{2+}$-FPP and an additional phosphate moiety bound only at the DHDDS active-site.

DHDDS can be subdivided into three domains: (i) an $\mathrm{N}$ terminal domain (NTD, residues 1-26), (ii) a canonical catalytic cis-prenyltransferase homology domain (residues 27-250), and (iii) a C-terminal domain (CTD, residues 251-333) (Supplementary Fig. 1). The catalytic cis-prenyltransferase homology domain, which forms the central region of DHDDS and serves for heterodimerization with $\mathrm{NgBR}$ (Fig. 2a, right), is composed of 7 $\alpha$-helices and $6 \beta$-strands (Supplementary Fig. 1), engulfing an elongated active-site cavity. This domain shares high homology with undecaprenyl diphosphate synthase (UPPS), a bacterial medium-chain cis-prenyltransferase homolog, with root-meansquare deviation (RMSD) $=0.76 \AA^{6}$ (Supplementary Figs. 5 and 6, Supplementary Data 1). The NTD and CTD flank the catalytic domain. The NTD is wrapped around the catalytic domain (Fig. 2a, left), with its single $\alpha$-helix, $\alpha \mathrm{N}$, directly packed against helix a7 via a network of hydrophobic interactions. Last, the CTD, absent from short- or medium-chain cis-prenyltransferases, features a "helix-turn-helix" motif composed of two consecutive helices situated immediately downstream of $\alpha 7$ (Fig. 2a, Supplementary Fig. 1). The first helix, $\mathrm{aC} 1$, is kinked by $30^{\circ}$ relative to the preceding $\alpha 7$ from the catalytic domain. The second helix, $\alpha \mathrm{C} 2$, is stabilized against $\mathrm{\alpha C} 1$ by a salt bridge between D273 and R306, and numerous electrostatic (E168-K320, E168-R321, D182-R309, and R196-E318) and hydrophobic interactions with the heterodimerization interface between DHDDS and NgBR (Fig. 2a).

Similar to DHDDS, the remnant N-terminal domain of $s \mathrm{NgBR}$ $\left(\mathrm{NTD}\right.$, residues $79^{*}-100^{*}$ ) also encompasses a single $\alpha$-helix, $\alpha \mathrm{N}$, 
likely serving as a structural link between the transmembrane and cytosolic domains in the intact protein. However, while NgBR was previously thought to share the canonical cis-prenyltransferase fold, similar to DHDDS ${ }^{10}$, the structure reveals that the two subunits share low structural similarity (RMSD $=2.07 \AA$, residues $100^{*}-293^{*}$ of sNgBR and $27-250$ of DHDDS). Indeed, the pseudo cis-prenyltranseferase homology domain of $\mathrm{sNgBR}$ encompasses only six $\alpha$-helices and five $\beta$-strands (Fig. 2a, Supplementary Fig. 1), in contrast to the seven $\alpha$-helices and six $\beta$-strands found in the other cis-prenyltransferases ${ }^{2,4}$. Moreover, $\mathrm{NgBR}$ and its yeast homology, Nus1, do not share high structural similarity $(\mathrm{RMSD}=1.33 \AA$ ), with the $\alpha 3$ of $\mathrm{NgBR}$ occupying the position of the antiparallel $\beta C-\beta C^{\prime}$ in Nus1 (Supplementary Fig. 5). The described fold of NgBR prevents interactions with substrates and hinders the catalytic activity of $\mathrm{NgBR}$, as specified below.

\section{Mechanism of tetramerization via a dimer-of-heterodimers} assembly. The DHDDS-sNgBR heterodimer is formed through a large interaction interface, with a buried surface area of $1938.0 \AA^{2}$ (Fig. 2a, right). This interface is mainly formed by helices $\alpha 5, \alpha 6$, and the $\beta E-\beta F$ linker of DHDDS and helices $\alpha 4, \alpha 5$, and the $\beta D-$ $\beta E$ linker of $\mathrm{NgBR}$, with an architecture reminiscent to that observed in homodimeric cis-prenyltransferases ${ }^{2}$. However, the heterodimeric interface also features contacts between the NgBR C-terminus and the active-site of DHDDS. Specifically, the structure reveals that the NgBR C-terminus, which encompasses the $\mathrm{RxG}$ motif and was previously suggested to play a critical role in hcis-PT activity, extends across the dimerization interface (Fig. 2a, left). This transverse interaction results in its direct involvement in the organization of the active-site of DHDDS and provides a structural framework for intersubunit communication.

While crystal packing analysis using the protein interfaces, surfaces and assemblies server ${ }^{24}$ suggested several possible assemblies, ranging from heterodimers to dodecamers, only one biological tetrameric assembly consistent with the oligomeric state in solution was detected. Importantly, this tetramer is formed by both homotypic interactions between DHDDS "helixturn-helix" motifs and heterotypic interactions of the "turn" region with $s \mathrm{NgBR}$ from adjacent ASUs, burying a total surface area of $793.3 \AA^{2}$ (Fig. 2b). Specifically, this interface is stabilized by two polar networks: (i) a salt-bridge network, centered at the interaction between R296 from the aC2 helix of one heterodimer and E264 and E271 from the $\mathrm{aC} 1$ helix of the adjacent heterodimer, and (ii) a hydrogen-bond network, originating from the interaction of D291, localized to the "turn" between $a \mathrm{C} 1$ and $\mathrm{aC}^{2}$, and Q268 from $\mathrm{aC} 1$, with $\mathrm{S}_{192}{ }^{*}$ of the adjacent heterodimer. Notably, this dimer-of-heterodimer arrangement is consistent with our cross-linking analysis (Fig. 1c).

Active-site organization. Although xcis-PT was crystallized in the presence of FPP and $\mathrm{Mg}^{2+}$, these substrates, along with an additional phosphate molecule, could only be detected in the active-site of DHDDS (Figs. 2a, 3, Supplementary Fig. 4). This active-site is formed by a superficial polar region, stabilizing the interaction with the pyrophosphate headgroups, and a deep hydrophobic tunnel accommodating the elongating carbon chain (Fig. 3a). The active-site contains two substrate-binding sites: an $\mathrm{S}_{1}$ site, which binds the initiatory substrate FPP, and an $\mathrm{S}_{2}$ site, which binds the IPP molecules used for chain elongation, and is occupied by the phosphate molecule in our structure. Importantly, the C-terminus of NgBR, encompassing the RxG motif, is directly involved in forming the superficial polar region of both sites.

At the $S_{1}$ site, the pyrophosphate moiety of FPP interacts with a $\mathrm{Mg}^{2+}$ ion (Fig. 3b, Supplementary Fig. 4). This $\mathrm{Mg}^{2+}$ ion, which plays a crucial role in pyrophosphate hydrolysis during the condensation reaction, is octahedrally coordinated by two of the pyrophosphate oxygens, three surrounding water molecules, and one carboxylate oxygen of the strictly conserved D34 (Fig. 3b, Supplementary Figs. 4 and 6, Supplementary Data 1$)^{2,4}$. In accordance with the key role of D34 and the spatial restraints of $\mathrm{Mg}^{2+}$ coordination, both the size and charge of aspartate at this position are crucial for catalysis, as substitution to $\mathrm{A}, \mathrm{E}$, or $\mathrm{N}$ resulted in $\sim 5-20$-fold reduction in catalytic activity $(1.32 \pm 0.09$, $0.35 \pm 0.07,0.10 \pm 0.01$, and $0.08 \pm 0.01 \mu \mathrm{mol} / \mathrm{h} / \mathrm{mg}$ protein for WT, D34A, D34E, and D34N, respectively; $n=5-7, P<0.001$ for each mutant) (Fig. 3f, Supplementary Fig. 7). In addition to the interaction with the $\mathrm{Mg}^{2+}$ ion, the pyrophosphate moiety is also directly stabilized by R37 and R38 from helix a1, and R85 from the $\beta \mathrm{B}-\alpha 3$ linker (Fig. 3b, Supplementary Fig. 4). Notably, the $\mathrm{R} 37 \mathrm{H}$ and $\mathrm{R} 38 \mathrm{H}$ mutations were previously associated with developmental epileptic encephalopathy (Fig. 3e). Indeed, these mutations confer an $\sim 5$-fold decrease in catalytic activity $(0.31 \pm$ $0.06,0.22 \pm 0.04 \mu \mathrm{mol} / \mathrm{h} / \mathrm{mg}$ protein for $\mathrm{R} 37 \mathrm{H}$ and $\mathrm{R} 38 \mathrm{H}$, respectively; $n=6, P<0.001$ for $\mathrm{R} 37 \mathrm{H}, n=5, P<0.01$ for $\mathrm{R} 38 \mathrm{H}$ ), in line with their role in FPP binding (Fig. 3f, Supplementary Figs. 4, 6, and 7, Supplementary Data 1).

While $\mathrm{xcis-PT}$ was crystallized in the absence of IPP, a phosphate molecule occupying the IPP pyrophosphate group position, as observed in other cis-prenyltransferases (Supplementary Fig. 8), is present at the $S_{2}$ site and is stabilized through a concerted coordination by the conserved R205, R211, and S213 (Fig. 3c, Supplementary Figs. 4 and 6, Supplementary Data 1) 2,4,23. The R211Q mutation was also associated with developmental epileptic encephalopathy (Fig. 3e). As expected, similar to $\mathrm{R} 37 \mathrm{H}$ and $\mathrm{R} 38 \mathrm{H}, \mathrm{R} 211 \mathrm{Q}$ exhibits an $\sim 5$-fold reduction in catalytic activity $(0.20 \pm 0.04 \mu \mathrm{mol} / \mathrm{h} / \mathrm{mg}$ protein; $n=5, P<0.001)$ (Fig. $3 \mathrm{f}$, Supplementary Figs. 4 and 7 ).

Importantly, the structure reveals that the RxG motif critically contributes to the formation of both $S_{1}$ and $S_{2}$ (Supplementary Fig. 4). At $S_{1}$, it stabilizes the $\beta B-\alpha 3$ linker of DHDDS by forming two polar interaction networks, interweaved between NgBR and DHDDS (Fig. 3c). One network is organized such that two saltbridge interactions, between E81 and R259*, and K84 and the carbonyl oxygen of $\mathrm{L} 291^{*}$, are anchored together by another saltbridge interaction between K84 and E81. The other network is formed by G292* backbone carbonyl interaction with N82 and R85, the latter involved in coordination of FPP (Fig. 3b). In addition, K293*, forming the C-terminal carboxylate group of $\mathrm{NgBR}$, interacts with both R37 and R85. At $\mathrm{S}_{2}$, the backbone nitrogen atoms of $\mathrm{L} 291^{*}$ and $\mathrm{G} 292^{*}$ directly coordinate the phosphate molecule (Fig. 3d). Importantly, in addition to the DHDDS-binding site mutants, $\mathrm{R} 290 \mathrm{H}^{*}$, localized to the $\mathrm{RxG}$ motif, was shown to result in a devastating congenital glycosylation disorder ${ }^{16}$. Situated between the $S_{1}$ and $S_{2}$ sites (Fig. 3e), this residue does not form a direct interaction with either the phosphate molecule or the FPP. However, inspection of other cisprenyltransferase structures, obtained in the absence of $\mathrm{Mg}^{2+}$, reveals that this conserved arginine can spatially and electrostatically replace the $\mathrm{Mg}^{2+}$ ion, transversely interacting with both the position corresponding to D34 and the pyrophosphate groups at $S_{1}$ and $S_{2}$ (Supplementary Fig. 9). Thus, R290H ${ }^{*}$ results in decreased catalytic activity $(0.91 \pm 0.19 \mu \mathrm{mol} / \mathrm{h} / \mathrm{mg}$ protein; $n=7$, $P<0.05$ ) (Fig. 3f, Supplementary Figs. 4 and 7). Together, our structure reveals the spatial clustering and functional convergence of disease mutations at the active-site, and provides a plausible explanation for the high conservation of the $\mathrm{RxG}$ motif due to its role in active-site organization.

Hydrophobic interactions in the active-site support isoprenoid chain elongation. According to the current model of chain 
a
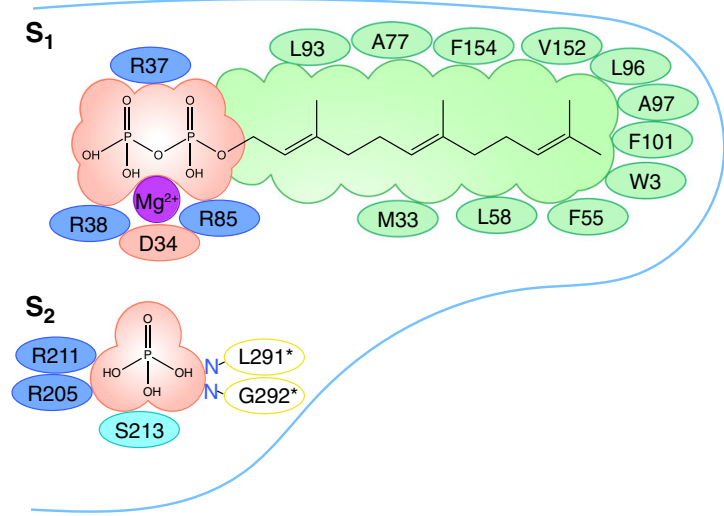

C

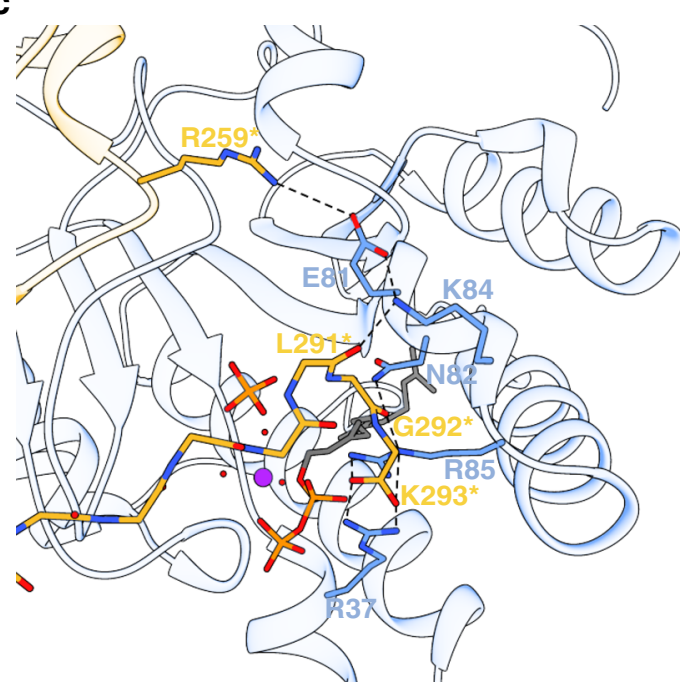

e

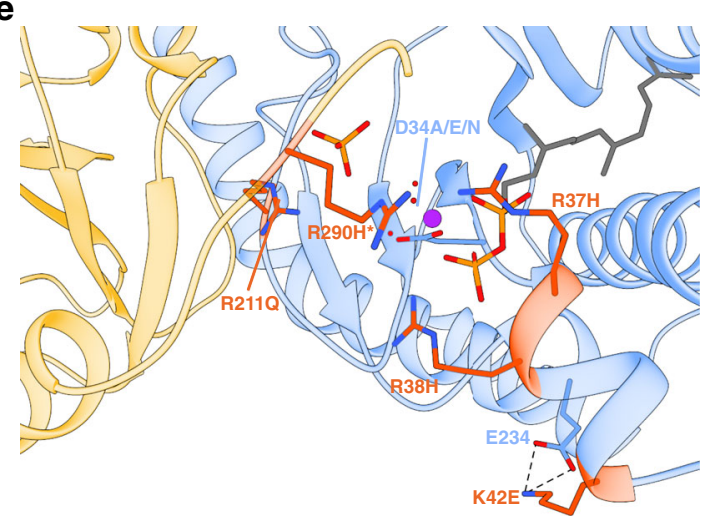

b

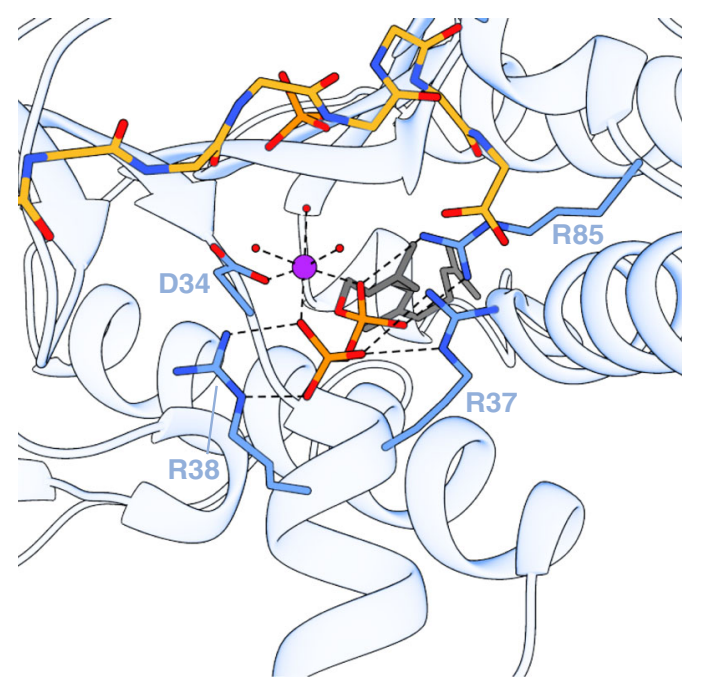

d

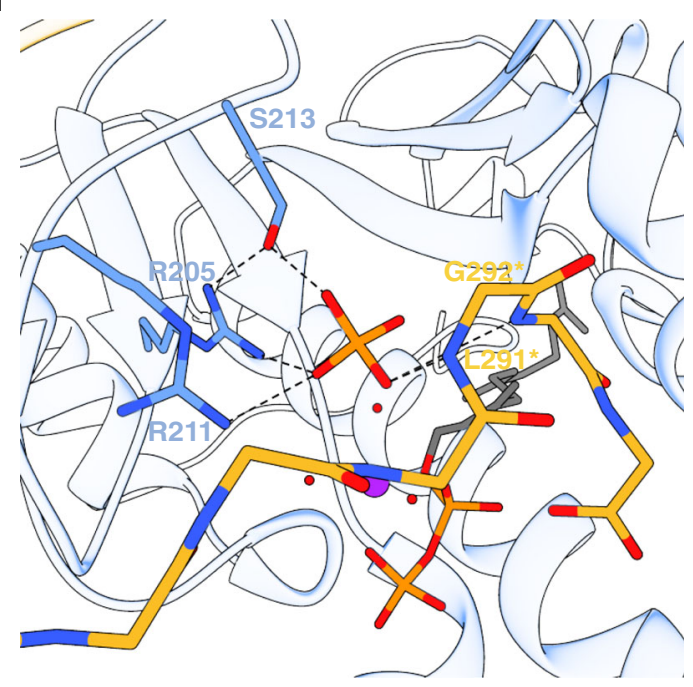

f

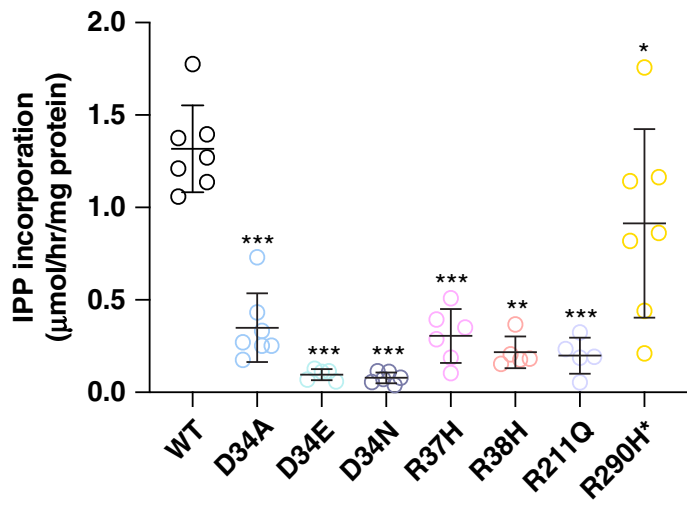

elongation by cis-prenyltransferases, the pyrophosphate headgroups are bound at the superficial polar region, while the carbon chains point toward the deep hydrophobic tunnel. During the catalytic cycle, the pyrophosphate group of the initiatory substrate at the $\mathrm{S}_{1}$ site (FPP, $\mathrm{C}_{15}$ ) is hydrolyzed, followed by condensation of the remaining carbons with the IPP $\left(C_{5}\right)$ from the $S_{2}$ site, yielding a 20-carbon polymer. Then, the elongated product translocates to the $S_{1}$ site, where the growing carbon chain permeates deeper into the hydrophobic tunnel of the active-site. Finally, a new IPP molecule binds to the $S_{2}$ site, and the cycle repeats until the active-site can no longer accommodate the longchain isoprenoid 25,26 .

The structure reveals that the hydrophobic tunnel of DHDDS is formed by $2 \alpha$-helices ( $\alpha 2$ and $\alpha 3)$ and $4 \beta$-strands $(\beta \mathrm{A}, \beta \mathrm{B}, \beta \mathrm{E}$, and $\beta \mathrm{F}$ ), similar to other cis-prenyltransferases ${ }^{26}$ (Fig. $\left.4 \mathrm{a}, \mathrm{b}\right)$. It was previously suggested that the opening between $\alpha 2$ and $\alpha 3$ 
Fig. 3 Structural organization of the active-site. a Two-dimensional interaction diagram of the $\mathrm{S}_{1}$ and $\mathrm{S}_{2}$ sites. Hydrophobic interactions, positively, and negatively charged DHDDS residues are colored green, blue, and red, respectively. $\mathrm{NgBR}$ residues interacting with the $\mathrm{S}_{2}$ site via their backbone nitrogen are indicated in yellow circles. b The FPP pyrophosphate-binding region at the $\mathrm{S}_{1}$ site. DHDDS is colored blue and $\mathrm{NgBR}$ is colored yellow. $\mathbf{c}$ Interactions of the NgBR C-terminal tail with the pyrophosphate-binding region of the $S_{1}$ site. DHDDS is colored blue and NgBR is colored yellow. $\mathbf{d}$ Phosphate coordination at the $\mathrm{S}_{2}$ site. $\mathrm{Mg}^{2+}$ and water molecules are shown as purple and red spheres, respectively. FPP and phosphate are shown as sticks. e Mapping of disease-associated mutations onto the xcis-PT heterodimer. Disease-associated positions are presented as sticks and colored orange. D34, mutated in this study, is shown as sticks. $\mathbf{f}$ In vitro activity of purified shcis-PT and mutants thereof was measured as IPP incorporation following 1-h incubation in the presence of $0.1 \mu \mathrm{M}$ enzyme, $20 \mu \mathrm{M}$ FPP, and $100 \mu \mathrm{M} \mathrm{IPP}$. Data are presented as mean \pm SEM. $n=5$ for D34E, R38H, and R211Q, $n=6$ for $\mathrm{D} 34 \mathrm{~N}$ and $\mathrm{R} 37 \mathrm{H}$, and $n=7$ for the WT, D34A, and R290H'. One-sided student's $t$ test was performed to compare all the mutants to the WT, except for $\mathrm{R} 38 \mathrm{H}$, for which one-sided Mann-Whitney test was performed. ${ }^{\star} P=0.04,{ }^{\star \star} P=0.0013,{ }^{\star \star \star} P<0.001$. Source data are provided as a Source Data file.
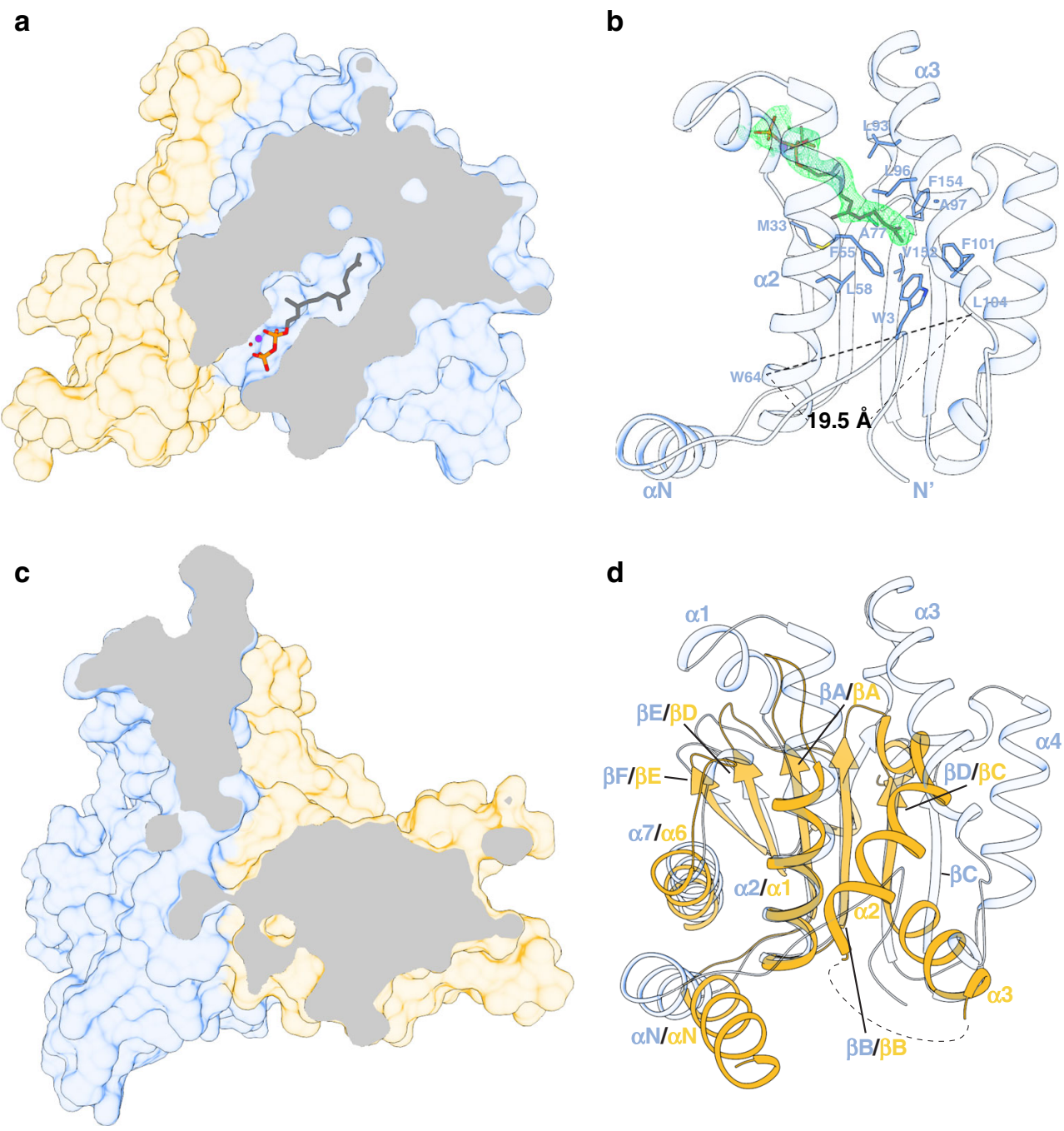

Fig. 4 The hydrophobic active-site tunnel. a Clipped surface representation of the heterodimer showing the FPP-binding site in DHDDS. DHDDS is colored blue and $\mathrm{NgBR}$ is colored yellow. $\mathrm{Mg}^{2+}$ and water molecules are shown as purple and red spheres, respectively. FPP is shown as sticks. b Coordination of the FPP tail within the active-site. The $2 F_{\mathrm{o}}-F_{\mathrm{c}}$ electron-density map of FPP, contoured at $\sigma=1$, is presented as a green mesh. FPP-interacting residues are shown as sticks. c Clipped surface representation of the heterodimer showing the lack of solvent-accessible cavity in NgBR. This view was obtained by superposition of $\mathrm{NgBR}$ onto DHDDS as viewed in panel (a). $\mathbf{d}$ Cartoon representation of a superposition between the cis-prenyltransferase homology domains of DHDDS and NgBR. The secondary structure elements are indicated. Note the tight packing of strands $\beta \mathrm{A}, \beta \mathrm{B}$, and $\beta \mathrm{D}$, and helices $\alpha 1$ and $\alpha 6$, leading to a collapse of the hydrophobic tunnel in NgBR, prohibiting FPP binding.

may be larger in DHDDS compared to short- and medium-chain cis-prenyltransferases, leading to a larger diameter enabling the accommodation of longer products ${ }^{26}$. Indeed, in DHDDS, the distance between $\alpha 2$ and $\alpha 3$, measured between the $\mathrm{C}_{\alpha}$ atoms of W64 and L104, is $19.5 \AA$ (Fig. 4b). In contrast, the distance between the corresponding positions in UPPS (F56 and E96), which synthesizes a 55-carbon isoprenoid, is only $15.3 \AA^{6}$.
Intriguingly, the structure shows that the unique NTD of DHDDS can snake into the binding site, interacting with the bound FPP molecule (Fig. 4b). Moreover, the conserved Nterminal W3 (Supplementary Fig. 6, Supplementary Data 1) interacts with F55, F101, and V152, thereby occluding the outlet of the hydrophobic tunnel of the active-site. This results in a surprisingly small active-site volume of $318 \AA^{3,27}$. Indeed, 
compared with the $371 \AA^{3}$ in the active-site of the medium-chain $\mathrm{UPPS}^{27}$, the xcis-PT site is seemingly inadequate for accommodating long-chain products. However, the high $\mathrm{B}$ factors of the NTD (Supplementary Fig. 10) suggest that it is mobile, possibly assuming different orientations relative to the active-site under physiological conditions. Indeed, it was suggested that hydrophobic residues (W12, F15, and I19) from helix aN interact with the membrane, resulting in increased catalytic activity in the presence of phospholipids ${ }^{23}$. Thus, we suggest that the NTD may shield the hydrophobic tunnel in the apo or FPP-bound states, while being expelled from the tunnel and interacting with the membrane upon chain elongation.

In addition to the active-site diameter, the composition of its terminal region also plays a key role in determination of product length. In UPPS, L137, localized to the N-terminus of $\beta \mathrm{D}$, was shown to be vital for determining chain length, with the L137A mutant increasing the product length from $\mathrm{C}_{55}$ to $\mathrm{C}_{75}{ }^{25}$. Our structure reveals that the corresponding position in DHDDS is replaced by $\mathrm{C} 148$, a hydrophilic and less bulky residue. Thus, C148 cannot occlude the hydrophobic tunnel outlet as efficiently as L137, similar to the L137A mutant, possibly contributing to long-chain product formation.

NgBR has been long known to lack catalytic activity of its own ${ }^{10}$. Nevertheless, the structural basis for this observation remained obscure. The structure clearly reveals that, in sharp contrast with DHDDS, sNgBR does not contain substrate-binding sites (Fig. 4c). Indeed, while the heterodimerization interface is structurally conserved (Fig. 2a), the NgBR region corresponding to the active-site in cis-prenyltransferases displays a significantly different structural arrangement (Fig. 4d). Specifically, strands $\beta A, \beta B$, and $\beta D$ and helices $\alpha 1$ and $\alpha 6$ are tightly packed via hydrophobic interactions, leaving this region without a detectable substrate-binding cavity and completely devoid of water molecules. This arrangement provides a structural explanation for the absence of $\mathrm{NgBR}$ catalytic activity, due to its incapacity to bind FPP and IPP. Thus, the only active-site of the complex is situated within the cis-prenyltransferase homology domain of DHDDS (Fig. 2).

The molecular mechanism of hcis-PT dysfunction in arRP. Disease mutations in DHHDS are clustered around the pyrophosphate-binding regions of the $S_{1}$ and $S_{2}$ sites (Fig. 3). Based on our structure, the functional perturbation caused by most disease-related positions (R37, R38, and R211) is straightforward, due to their direct involvement in substrate binding. However, the effect of K42E $(0.83 \pm 0.14 \mu \mathrm{mol} / \mathrm{h} / \mathrm{mg}$ protein; $n=$ 7, $P<0.01$ ) (Figs. 3e and 5a, Supplementary Fig. 7), a mutation leading to isolated retinitis pigmentosa ${ }^{12,13}$, is not as obvious. Careful examination of K42 surroundings revealed that it forms a salt bridge with E234 (Figs. 3e and 5b). Thus, we hypothesized that the functional effect of the K42E mutation is relayed to the active-site via an alternative interaction with adjacent positively charged active-site residues.

To test this hypothesis, we performed an all-atom MD simulation of shcis-PT encompassing DHDDS-WT or DHDDS-K42E (Fig. 5, Supplementary Fig. 11), in the absence of substrates. We calculated three independent 100-ns trajectories for the WT complex and four independent 100-ns trajectories for the mutant complex (Supplementary Fig. 11). Close inspection of the surroundings of position 42 revealed that while the K42-E234 salt bridge remains stable through the simulation (Fig. 5b, d, Supplementary Fig. 11), K42E results in the formation of a new stable salt bridge with the catalytic residue R38 (Fig. 5c, e, Supplementary Fig. 11). Indeed, the distance distributions of positions $42-234$ and 38-42 are inversely correlated. Specifically, while the WT protein displays a narrow distribution of short distances between positions 42 and 234, introduction of K42E leads to a wider distribution over longer distances, as expected due to the charge repulsion (Fig. 5d). Conversely, while K42E displays a narrow distribution of short distances between positions 38 and 42 , these positions display a wider distribution of longer distances in the context of the WT protein, as expected due to their similar charges (Fig. 5e). Importantly, despite the presence of adjacent positively charged residues (e.g., R37 and K41), E42 forms a stable salt bridge specifically with R38 (Supplementary Fig. 12).

In addition, we examined the effect of K42E on the dynamics of the entire active-site by monitoring active-site residues within $5 \AA$ of the crystallized substrates (Fig. $5 \mathrm{f}$ ). We noticed that within the timeframe of the simulations, the K42E mutation results in active-site destabilization, including the C-terminus of $\mathrm{NgBR}$, as reflected by the overall increase in RMSD (representing the deviation of the coordinates relative to the initial state of the simulation) in three of four simulations (Supplementary Fig. 11), and by the overall increase in RMSF (representing the fluctuation of the residue side chain relative to its average position along the simulation) (Fig. 5f, Supplementary Fig. 11). These results support the notion that $\mathrm{K} 42 \mathrm{E}$ underlies the formation of aberrant polar networks, hindering the capacity of active-site residues to bind substrates, thereby reducing the catalytic activity.

\section{Discussion}

Here, we provide the structure of a heteromeric cis-prenyltransferase. Our biochemical and structural analyses of hcis-PT reveal a heterotetrameric assembly, formed via a dimerof-heterodimers mechanism, mainly through homotypic interface formation between the CTD in DHDDS (Fig. 2). Furthermore, the structure elucidates the molecular determinants governing substrate binding, the contribution of the RxG motif of $\mathrm{NgBR}$ to active-site formation by transverse interactions with DHDDS, and the effect of active-site resident disease mutations (Fig. 3). In addition, the structure unveils how the architecture of NgBR precludes its endogenous catalytic activity while enabling intersubunit communication, and highlights the molecular determinants supporting formation of long-chain isoprenoid (Fig. 4). Finally, using MD simulations, we unveil the molecular mechanisms of a retinitis pigmentosa causing mutation in hcisPT (Fig. 5).

To date, dimerization is considered as the common tertiary organization of cis-prenyltransferases ${ }^{2,4,8,22}$. Additionally, the structurally characterized family members were homodimeric, encompassing an active-site within each subunit, and thus exhibiting an overall functional symmetry. Recently, the presence of a single heterodimer in the crystallographic ASU of hcis-PT in complex with IPP was interpreted as an evidence for its heterodimeric stoichiometry ${ }^{23}$. Nevertheless, the elution profile reported is identical to that we present here, suggesting the presence of a common heterotetrameric assembly (Fig. 1). Moreover, we show here using SEC-MALS, native ESI-MS, and cross-linking (Fig. 1) that shcis-PT exhibits a heterotetrameric organization, achieved via a dimer-of-heterodimers assembly mediated by homotypic interactions of the CTD of DHDDS (Fig. 2). Importantly, intact hcis-PT harboring the transmembrane domain of NgBR, expressed and purified from Expi293F cells, was previously subjected to size-exclusion analysis. Although its mass was not directly assessed, it also shares a markedly similar elution profile with shcis- $\mathrm{PT}^{21}$. This tetrameric assembly mode may contribute to the previously suggested mutual stabilizing effect resulting from NgBR and DHDDS co-expression in cells ${ }^{10}$. Interestingly, a deletion mutant of DHDDS lacking the CTD does not support 
a

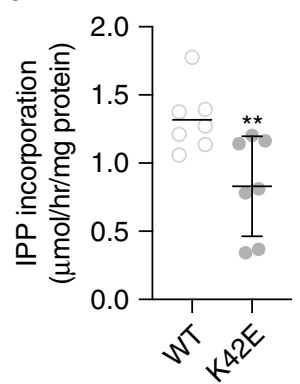

b

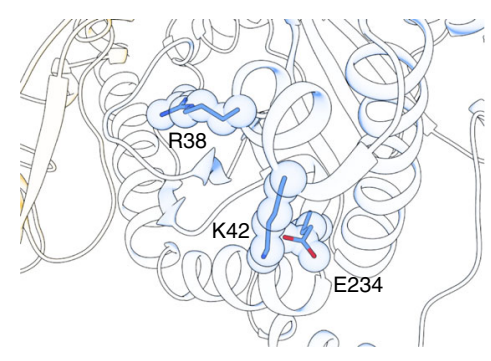

C

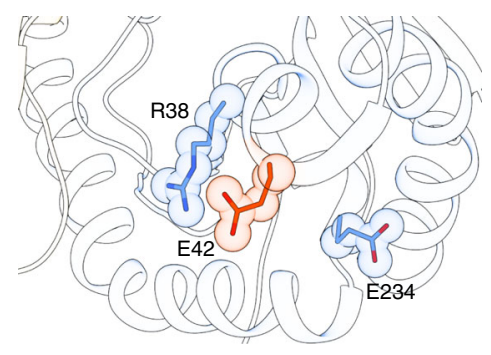

d

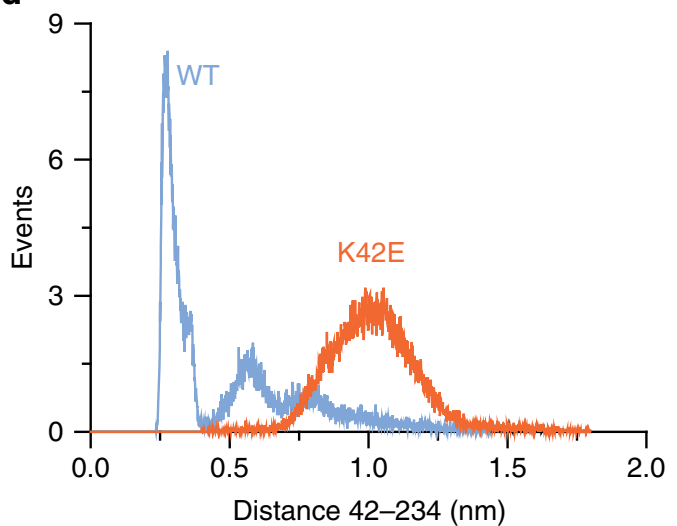

e

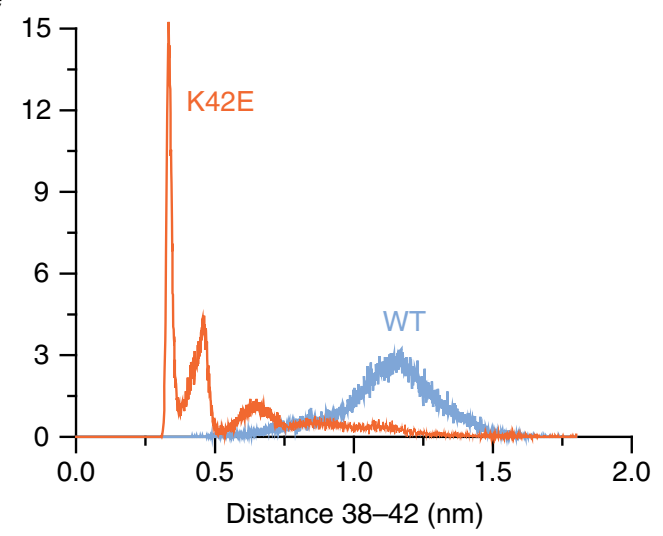

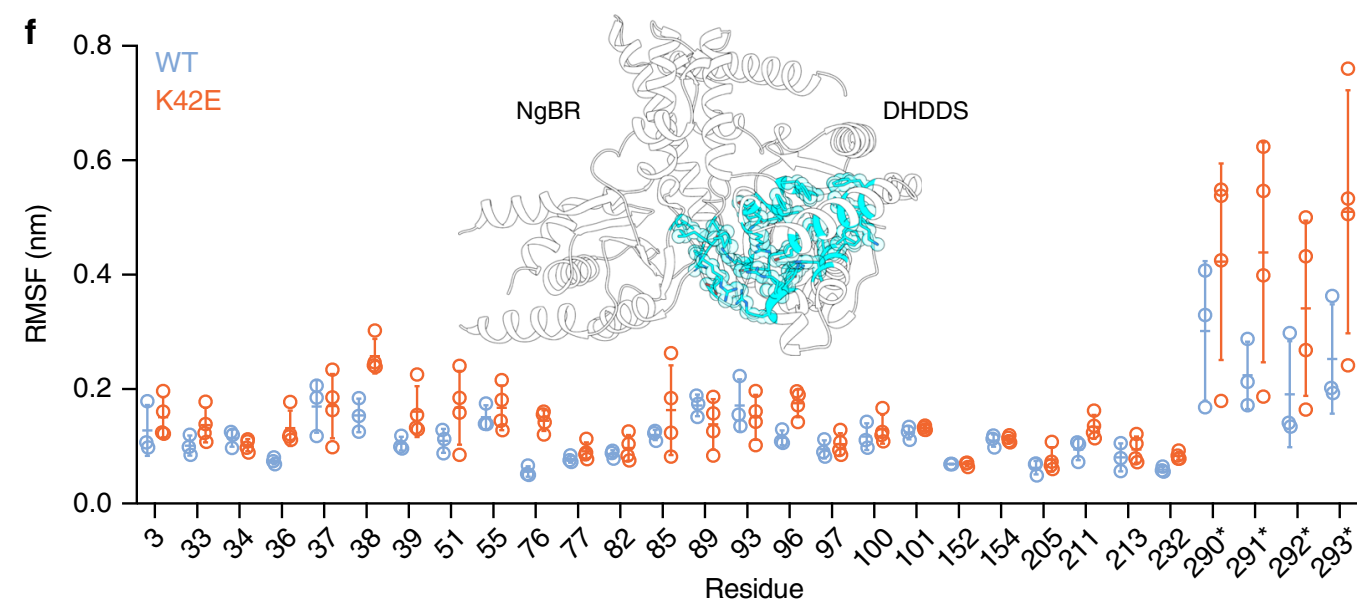

Fig. 5 Structural basis for hcis-PT-related arRP. a In vitro activity of purified shcis-PT harboring the K42E mutation was measured as IPP incorporation following 1-h incubation in the presence of $0.1 \mu \mathrm{M}$ enzyme, $20 \mu \mathrm{M}$ FPP, and $100 \mu \mathrm{M}$ IPP. Data are presented as mean \pm SEM, $n=7$ independent experiments. The activity of the WT is shown for reference. One-sided student's $t$ test was performed for data analysis, ${ }^{\star \star} P=0.0069$. Source data are provided as a Source Data file. b A representative conformation of the most abundant cluster of the WT protein. R38, K42, and E234 are shown as sticks. R38 points toward the active-site cavity, while $\mathrm{K} 42$ and E234 form a stable salt bridge. c A representative conformation of the most abundant cluster of the complex harboring DHDDS-K42E. R38, E42, and E234 are shown as sticks. R38 points away from the active-site cavity, forming a new salt bridge with the mutant E42, colored orange. d Distance distribution between positions 42 and 234, measured between the charge centers of E234 and K42 (light blue) or E42 (orange). e Distance distribution between positions 38 and 42, measured between the charge centers of R38 and K42 (light blue) or E42 (orange). f Average ( \pm SD) RMSF of the active-site residues within $5 \AA$ of the crystallized substrates for the WT (light blue, $n=3$ ) and mutant (orange, $n=4$ ) complexes. Source data are provided as a Source Data file. Inset: Active-site residues within $5 \AA$ of the crystallized substrates are colored cyan and shown as sticks.

cell growth in a yeast complementation assay ${ }^{23}$, underscoring the functional significance of tetramerization.

Our structure hints toward the mechanisms allowing longchain isoprenoid synthesis by his-PT. While the deep hydrophobic tunnel, engulfing the elongating product, is walled by $\alpha 2$, $\alpha 3, \beta \mathrm{A}, \beta \mathrm{B}, \beta \mathrm{E}$, and $\beta \mathrm{F}$ (Fig. 4), previous studies pinpointed the length of $\alpha 3$ as a key contributor to chain-length determination ${ }^{26}$.
Indeed, previous studies of UPPS showed that an insertion of 3 residues, corresponding to ${ }^{107} \mathrm{EKE}^{109}$ in human DHDDS, led to an increase in product length from $\mathrm{C}_{55}$ to $\mathrm{C}_{70}$. The product length was further increased to $\mathrm{C}_{75}$ if a 5-residue insertion, mimicking the yeast ortholog Srt1, was introduced, establishing the correlation between the length of $\alpha 3$ and the product ${ }^{26}$. Finally, deletion of the ${ }^{107} \mathrm{EKE}^{109}$ sequence was shown to result in product 
shortening ${ }^{23}$. In full agreement with these observations, the structure of xcis-PT reveals that the ${ }^{107} \mathrm{EKE}^{109}$ results in a kink in a3, leading to an $\sim 4 \AA$ increase in the hydrophobic tunnel diameter (Fig. 4b) compared with UPPS. In addition to the increased tunnel diameter, we propose that the local environment conferred by C148 may allow expulsion of the elongating product directly into the adjacent membrane during catalysis, allowing the formation of products that exceed the active-site volume. Together, the increased length of a3, the composition of the hydrophobic tunnel outlet, and the membrane association of the complex jointly contribute to long-chain isoprenoid production by hcis-PT.

The organization of the CTD of DHDDS, underlying hcis-PT tetramerization, provides a mechanistic explanation for the poor activity exhibited by DHDDS homodimers ${ }^{20}$. The helix-turnhelix motif, following a7 (Fig. 2), is incompatible with formation of the extensive interaction network observed within each hcis-PT heterodimer active-site (Fig. 3). In contrast, the assembly of DHDDS with $\mathrm{NgBR}$ allows the complementation of the activesite by the transverse interactions with the C-terminal tail of $\mathrm{NgBR}$ (Fig. 3). Importantly, such transverse interactions are observed also in homodimeric cis-prenyltransferases (Supplementary Fig. 9), lacking a C-terminal helix-turn-helix motif, supporting the functional importance of these interactions in coupling active-site organization with enhanced catalytic activity. Consistent with this notion, mutations in the C-terminal tail of $\mathrm{NgBR}$ were previously shown to result in reduced catalytic activity $^{16,21}$. Now, our structure offers a mechanistic understanding of the effects imposed by these mutations. Indeed, introduction of $\mathrm{R} 290 \mathrm{H}^{*}$ in the context of the intact complex was shown to result in decreased catalytic activity ${ }^{16,21}$. The soluble construct we used for structural investigation also exhibits decreased activity upon mutation (Fig. 3). Structural comparison to other cis-prenyltransferases (Supplementary Fig. 9) revealed that the guanidinium group of $\mathrm{R} 290^{*}$ is necessary and sufficient to substitute the $\mathrm{Mg}^{2+}$ ion within the active-site, interacting with both substrates. This interaction may contribute to the translocation of the product pyrophosphate group from $S_{2}$ to $S_{1}$ following the condensation reaction and release of the hydrolyzed pyrophosphate and $\mathrm{Mg}^{2+}$ ion ${ }^{25,26}$. In contrast, histidine is both shorter and partially charged under physiological $\mathrm{pH}$, making this residue insufficient to functionally and structurally substitute for the conserved arginine ${ }^{28}$. Moreover, further emphasizing the functional significance of the $\mathrm{NgBR} \mathrm{C}$-terminus, previous introduction of the G292A* mutation increased the $K_{\mathrm{M}}$ for IPP by $\sim 6$ fold while reducing the turnover rate by $\sim 12$-fold ${ }^{21}$. As shown here, G292* is directly involved in IPP binding (Fig. 3), and measuring the $\varphi / \psi$ angles reveals that this position can only be occupied by glycine ${ }^{29}$. Finally, we show that the backbone carboxylate of $\mathrm{K}_{293}{ }^{*}$ interacts with the catalytic residues R37 and R85 (Fig. 3c). In accordance with the pivotal role of this interaction network, either deletion of K293 or the addition of a terminal alanine resulted in diminished catalytic activity of the intact complex ${ }^{21}$. Together, the heterodimerization architecture observed here supports the notion that although DHDDS is considered as the catalytically active subunit, both subunits are necessary for efficient dolichol synthesis.

By forming the hcis-PT complex, DHDDS and NgBR were shown to play a crucial role in cellular dolichol synthesis ${ }^{10,30}$. Mapping disease mutations in DHDDS onto the structure (Figs. 3 and 5) reveals their clustering around the pyrophosphate-binding regions of the $S_{1}$ and $S_{2}$ sites (Fig. 5). Interestingly, these mutations can be subdivided into mutations that directly or indirectly interfere with substrate association. The mutations that directly perturb substrate binding include R37H, R38H, and R211Q (Fig. 3e). These mutations result in a similar reduction in catalytic activity (Fig. 3f) and are clinically associated with developmental epileptic encephalopathies ${ }^{14}$. In contrast, K42E, which leads to isolated $\operatorname{arRP} \mathrm{P}^{12,13}$, is indirectly involved in substrate coordination (Fig. 3e) and leads to a milder reduction in catalytic activity (Fig. 5a). As the pathogenic effect of K42E was not readily apparent from the structure, we hypothesized that the charge reversal caused by K42E may alter the electrostatic interactions that play a vital role in substrate coordination (Figs. 3e and 5). Remarkably, MD simulations uncovered a novel salt bridge between the mutant E42 and the catalytic residue R38 (Fig. 5b-e), hindering substrate binding, and thus providing a mechanistic explanation for the pathogenicity of this mutation (Fig. 5a). Interestingly, additional to this local structural alteration, we also observed a tendency for global increase in active-site dynamics, including the C-terminus of NgBR (Fig. 5f, Supplementary Fig. 11). Inspection of the active-site residues RMSD along the trajectories of the individual simulations revealed destabilization in three of the four replicates (Supplementary Fig. 11c), and the mean RMSF values of these residues are consistently slightly higher in the mutant compared to the WT complex (Fig. 5f). This allosteric effect, emanating from the charge reversal in position 42 and displacement of R38, aligns well with the observation that patients harboring the K42E mutation display a characteristic shortening of their plasma and urinary dolichols ${ }^{31}$. Specifically, we suggest that the globally enhanced dynamics of the active-site may result in weakening of the association with long-chain products, leading to their premature release. Future studies are needed to determine whether altered substrate interaction, either through direct or indirect mechanisms, converges into a similar enhancement of active-site dynamics, resulting in a common outcome of product-length shortening, along with the reduction in catalytic activity (Figs. $3 \mathrm{f}$ and $5 \mathrm{a}$ ).

Together, with the growing spectrum of diseases related to hcis-PT dysfunction, the structure sheds light on the mechanisms of dolichol synthesis and their disruption in disease. Moreover, it establishes a molecular framework that may enable the rational design of specific hcis-PT activity modulators for the treatment of arRP and additional congenital glycosylation disorders.

\section{Methods}

Cloning. Full-length human DHDDS (residues 1-333, UniProt Q86SQ9) was cloned into pET-32b plasmid and sNgBR (residues $73^{*}-293^{*}$, UniProt Q96E22) or sNgBR $\Delta 167^{*}-175^{*}$ were cloned into pETM-11 plasmid as thioredoxin (TRX) fusion proteins ${ }^{32}$. The constructs include a $6 \times$ His-tag (DHDDS) or Strep-tag $\left(\mathrm{sNgBR} / \mathrm{sNgBR} \Delta 167^{*}-175^{*}\right)$ to facilitate protein purification and a TEV-protease (tobacco etch virus) cleavage site to remove the affinity tags and TRX fusion. Mutations were introduced using the QuickChange method and verified by sequencing. Primers used for mutagenesis are listed in Supplementary Table 1.

Protein expression and purification. Escherichia coli T7-expressed competent cells were co-transformed with DHDDS and $\mathrm{sNgBR}$ (shcis-PT) or sNgBR $\Delta 167^{*}-175^{*}$ (xcis-PT), grown in Terrific Broth medium at $37^{\circ} \mathrm{C}$ until reaching $\mathrm{OD}_{600 \mathrm{~nm}}=0.6$, and induced at $16^{\circ} \mathrm{C}$ by adding $0.5 \mathrm{mM}$ isopropyl $\beta$-D-1 thiogalactopyranoside (IPTG). Proteins were expressed at $16^{\circ} \mathrm{C}$ for $16-20 \mathrm{~h}$, harvested by centrifugation $(\sim 5700 \times g$ for $15 \mathrm{~min})$, and then resuspended in a lysis buffer containing $20 \mathrm{mM} 4$-(2-hydroxyethyl)-1-piperazineethanesulfonic acid (HEPES), pH 7.5, $150 \mathrm{mM} \mathrm{NaCl}$, and $1 \mathrm{mM}$ tris(2-carboxyethyl)phosphine (TCEP) and $0.02 \%(\mathrm{w} / \mathrm{v})$ triton X-100, supplemented with $1 \mu \mathrm{g} / \mathrm{ml}$ DNase I and a protease inhibitor mixture. Resuspended cells were homogenized and disrupted in a microfluidizer. Soluble proteins were recovered by centrifugation at $\sim 40,000 \times g$ for $45 \mathrm{~min}$ at $4^{\circ} \mathrm{C}$. Overexpressed proteins were purified on a HisTrap HP column, followed by purification on a Strep-Tactin column and TEV-protease cleavage of the purification tags and TRX fusions. The reaction mixture was concentrated and loaded onto a Superdex-200 preparative size-exclusion column pre-equilibrated with $20 \mathrm{mM}$ HEPES, pH 7.5, $150 \mathrm{mM} \mathrm{NaCl}$, and $1 \mathrm{mM}$ TCEP. Purified proteins were flash-frozen in liquid nitrogen and stored at $-80^{\circ} \mathrm{C}$ until use. Protein purity was $>95 \%$, as judged by SDS-PAGE.

Cross-linking. About $8 \mu \mathrm{M}$ of shcis-PT were cross-linked by incubation with $0.005 \%$ glutaraldehyde at room temperature for $15 \mathrm{~min}$. Reactions were quenched by the addition of sodium dodecyl sulfate and $\beta$-mercaptoethanol containing 
Table 1 Data collection and refinement statistics.

6Z1N: xcis-PT

\section{Data collection}

Space group

Cell dimensions

$a, b, c(\AA)$

$\alpha, \beta, \gamma\left({ }^{\circ}\right)$

Resolution $(\AA)$

$R_{\text {sym }}$ or $R_{\text {merge }}$

$|/ \sigma|$

Completeness (\%)

Redundancy

Refinement

Resolution ( $\AA$ )

No. of reflections

$R_{\text {work }} / R_{\text {free }}$

No. of atoms

Protein

Ligand/ion

Water

$B$ factors

Protein

Ligand/ion

Water

R.m.s. deviations

Bond lengths $(\AA)$

Bond angles $\left({ }^{\circ}\right)$

Note: Statistics are based on one crystal per dataset.

*Values in parentheses are for the highest-resolution shel.

sample buffer, followed by $10 \mathrm{~min}$ of incubation at room temperature. Cross-linked products were analyzed by SDS-PAGE.

ESI-MS. shcis-PT $(20 \mu \mathrm{M})$ was transferred into $200 \mathrm{mM}$ ammonium acetate, $\mathrm{pH}$ 7.5 , by Zeba Spin columns $(0.5 \mathrm{~mL}, 7-\mathrm{kDa}$ cutoff $)$ and adjusted to $10 \mu \mathrm{M}$ concentration. The sample was loaded into a homemade quartz-glass ESI tip that was mounted onto a custom-built nESI source interfaced to Waters Synapt G2Si. Analyses at different activation settings were performed. Low activation (trap collision energy $10 \mathrm{~V}$ ) was used to obtain native-like conditions, while high-trap collisional energies (up to $110 \mathrm{~V}$ ) were employed to strip the adducts and obtain more accurate mass. Key instrument parameters were sampling cone voltage $40 \mathrm{~V}$, source offset $20 \mathrm{~V}$, trap gas $4 \mathrm{ml} / \mathrm{min}$ and source temperature $20^{\circ} \mathrm{C}$, and ESI tip voltage $1.8 \mathrm{kV}$. Data were analyzed in MassLynx 4.1 .

Enzyme kinetics. The activity of purified shcis-PT was measured using a radioligand-based assay $20,32,33$. About $0.01-0.1 \mu \mathrm{M}$ of purified proteins were mixed with FPP and $\left[{ }^{14} \mathrm{C}\right]-\mathrm{IPP}$ to initiate the reaction in buffer composed of $25 \mathrm{mM}$ Tris$\mathrm{HCl}, \mathrm{pH} 7.5,150 \mathrm{mM} \mathrm{NaCl}, 10 \mathrm{mM} \beta$-mercaptoethanol, $0.02 \%$ Triton X-100, and $0.5 \mathrm{mM} \mathrm{MgCl}_{2}$ at $30^{\circ} \mathrm{C} .15 \mathrm{mM}$ EDTA (final concentration) was added to quench the reaction, and $500 \mu \mathrm{L}$ of water-saturated 1-butanol was added to extract the reaction products by thorough vortexing. Initial rates were measured by quenching the reaction at $10 \%$ or lower substrate consumption. The products, encompassing ${ }^{14} \mathrm{C}$, were quantitated using a scintillation counter. The $K_{\mathrm{M}}$ value of FPP was determined by varying [FPP] while holding [IPP] constant at $100 \mu \mathrm{M}$, and the $K_{\mathrm{M}}$ value of IPP was determined by varying [IPP] while holding [FPP] constant at $10 \mu \mathrm{M}$. Kinetic constants were obtained by fitting the data to the Michaelis-Menten equation using Origin 7.0 (OriginLab, USA). For mutant analysis, $0.1 \mu \mathrm{M}$ of purified proteins were mixed with $20 \mu \mathrm{M}$ FPP and $100 \mu \mathrm{M}\left[{ }^{14} \mathrm{C}\right]-\mathrm{IPP}$, and the reaction was quenched after $1 \mathrm{~h}$ followed by product extraction and quantitation. The Shapiro-Wilk test of normality was performed using Graphpad Prism 8. Several batches of proteins were tested to ensure that the mutational effect is reproducible compared to the WT. One-sided student's $t$ test and the Mann-Whitney test were used for analysis of normally and non-normally distributed data, respectively, using Graphpad Prism 8.

Crystallization and structure determination. Initial crystallization screens were performed using $\sim 15 \mathrm{mg} / \mathrm{mL}$ purified xcis-PT in the presence of $0.5 \mathrm{mM} \mathrm{MgCl}$ and $760 \mu \mathrm{M} \mathrm{FPP}$ at $19^{\circ} \mathrm{C}$ using the sitting-drop vapor-diffusion method. Crystals were obtained in $0.1 \mathrm{M} \mathrm{NaCl}, 0.1 \mathrm{M} \mathrm{NaPO}_{4}$, pH 7.0, and 33\% w/v PEG 300. Data were collected at $100 \mathrm{~K}$ using a wavelength of $0.976 \AA$ at the Diamond Light Source (DLS, Oxfordshire, United Kingdom) beamline I03. Data collection was performed using the Generic Data Acquisition software on-site. Integration, scaling, and merging of the diffraction data were done with the XDS program ${ }^{34}$. The structure was solved by automated molecular replacement and initial model building using the programs MrBump ${ }^{35}$ and $\mathrm{CCP}^{36}$ (Table 1). Iterative model building and refinement were carried out in PHENIX ${ }^{37}$ with manual adjustments using COOT $^{38}$. Ramachandran analysis was performed using MolProbity ${ }^{39}$. About $97.12 \%$ and $2.88 \%$ of residues were in the Ramachandran favored and allowed regions, respectively. Structural illustrations were prepared with UCSF Chimera (https://www.cgl.ucsf.edu/chimera).

Conservation analysis of DHDDS. The Ensembl server ${ }^{40}$ was used to search for human DHDDS orthologs. These sequences were used to generate a multiplesequence alignment using Clustalw ${ }^{41}$. The resulting alignment was used as input for the Consurf server ${ }^{42}$, which outputs a conservation score for each residue.

Molecular dynamics (MD). A single human NgBR-DHDDS heterodimer was used. Mutation K42E was modeled using Schrodinger's Maestro 11.2. All the structures were prepared using the Protein Preparation Wizard (Schrödinger Release 2017-2: Schrödinger Suite 2019-2 Protein Preparation Wizard; Schrödinger, LLC, New York, NY, 2016) as implemented in Schrodinger's Maestro 11.2 (Schrödinger Release 2017-2: Schrödinger Suite 2019-2 Protein Preparation Wizard; Schrödinger, LLC, New York, NY, 2016). This protocol adds missing hydrogen atoms considering a $\mathrm{pH}$ value of $7.0 \pm 1.0$, optimizes the hydrogen-bond network, and performs restrained minimization. Crystallographic ligands, ions, and water were removed. The MD simulations were performed using GROMACS version $2018.2^{43}$, with OPLS forcefield ${ }^{44}$. Each protein complex was submerged in TIP3P water model in a triclinic box with $15 \AA$ extension around the protein. Potassium and chloride ions were added to the water phase in order to neutralize the system and to obtain a salt concentration of $0.15 \mathrm{M}$. The simulations were conducted in periodic boundary conditions with particle-mesh Ewald electrostatics with $10 \AA$ cutoff for long-range interactions ${ }^{45}$. First, the simulated systems were energy minimized with the steepest descent minimization algorithm in order to remove van der Waals clashes. Afterward, two equilibration steps were used: $100 \mathrm{ps}$ of simulation in NVT ensemble followed by 100 ps of NPT ensemble, where the heavy atoms of the protein are restrained in both steps. During the equilibration stages, an integration time step of $1 \mathrm{fs}$ was used. Finally, the production simulations were carried out for $100 \mathrm{~ns}$ with a constant temperature of $300 \mathrm{~K}$ under V-rescale coupling algorithm and constant pressure of 1 atm under Parrinello-Rahman coupling algorithm. The LINCS algorithm was applied to bond lengths involving hydrogen, allowing an integration time step of $2 \mathrm{fs}^{44}$. Three and four replicas of MD simulations were conducted for each of the WT and mutant constructs, respectively. The resulting trajectories were visually inspected using VMD 1.9.3 software s6 $^{4}$. Clustering was performed by the clustering analysis tool of Gromacs (gmx cluster). The GROMOS clustering algorithm with a cutoff of 0.12 $\mathrm{nm}$ was used to determine the neighboring structures in the clusters. The stability of the resulting trajectories and the average mobility of the protein residues $5 \AA$ around the crystallographic ligand were tested based on the RMSD of the backbone atoms of the protein from the equilibrated structures and on the RMSF, respectively. RMSD and RMSF were calculated using the rms and rmsf utilities of the GROMACS package, respectively. Distances along the trajectories' time and distances probabilities were calculated using the distance utility.

Reporting summary. Further information on research design is available in the Nature Research Reporting Summary linked to this article.

\section{Data availability}

Atomic coordinates and structure factors for the structure of $\mathrm{xcis- \textrm {PT }}$ in complex with $\mathrm{Mg}^{2+}$ and FPP have been deposited in the Protein Data Bank with accession number $6 \mathrm{Z} 1 \mathrm{~N}$. Source data are provided with this paper.

Received: 22 June 2020; Accepted: 23 September 2020; Published online: 19 October 2020

\section{References}

1. Grabińska, K. A., Park, E. J. \& Sessa, W. C. CIS-prenyltransferase: new insights into protein glycosylation, rubber synthesis, and human diseases. J. Biol. Chem. 291, 18582-18590 (2016).

2. Takahashi, S. \& Koyama, T. Structure and function of cis-prenyl chain elongating enzymes. Chem. Rec. 6, 194-205 (2006).

3. Ogura, K. \& Koyama, T. Enzymatic aspects of isoprenoid chain elongation. Chem. Rev. 98, 1263-1276 (1998).

4. Chen, C. C. et al. Versatile cis-isoprenyl diphosphate synthase superfamily members in catalyzing carbon-carbon bond formation. ACS Catal. 10, 4717-4725 (2020).

5. Yamashita, S. et al. Identification and reconstitution of the rubber biosynthetic machinery on rubber particles from Hevea brasiliensis. Elife 5, e19022 (2016). 
6. Guo, R. T. et al. Crystal structures of undecaprenyl pyrophosphate synthase in complex with magnesium, isopentenyl pyrophosphate, and farnesyl thiopyrophosphate: roles of the metal ion and conserved residues in catalysis. J. Biol. Chem. 280, 20762-20774 (2005).

7. Ko, T. P. et al. Substrate-analogue complex structure of Mycobacterium tuberculosis decaprenyl diphosphate synthase. Acta Crystallogr. Sect. F Struct. Biol. Commun. 75, 212-216 (2019).

8. Fujihashi, M. et al. Crystal structure of cis-prenyl chain elongating enzyme, undecaprenyl diphosphate synthase. Proc. Natl Acad. Sci. USA 98, 4337-4342 (2001).

9. Wang, W. et al. The structural basis of chain length control in Rv1086. J. Mol. Biol. 381, 129-140 (2008).

10. Harrison, K. D. et al. Nogo-B receptor is necessary for cellular dolichol biosynthesis and protein N-glycosylation. EMBO J. 30, 2490-2500 (2011)

11. Schwarz, F. \& Aebi, M. Mechanisms and principles of $\mathrm{N}$-linked protein glycosylation. Curr. Opin. Struct. Biol. 21, 576-582 (2011).

12. Zelinger, L. et al. A missense mutation in DHDDS, encoding dehydrodolichyl diphosphate synthase, is associated with autosomal-recessive retinitis pigmentosa in ashkenazi jews. Am. J. Hum. Genet. 88, 207-215 (2011).

13. Züchner, S. et al. Whole-exome sequencing links a variant in DHDDS to retinitis pigmentosa. Am. J. Hum. Genet. 88, 201-206 (2011).

14. Hamdan, F. F. et al. High rate of recurrent de novo mutations in developmental and epileptic encephalopathies. Am. J. Hum. Genet. 101, 664-685 (2017).

15. Sabry, S. et al. A case of fatal type I congenital disorders of glycosylation (CDG I) associated with low dehydrodolichol diphosphate synthase (DHDDS) activity. Orphanet J. Rare Dis. 11, 84 (2016).

16. Park, E. J. et al. Mutation of Nogo-B receptor, a subunit of cisprenyltransferase, causes a congenital disorder of glycosylation. Cell Metab. 20, 448-457 (2014)

17. Guo, J. F. et al. Coding mutations in NUS1 contribute to Parkinson's disease. Proc. Natl Acad. Sci. USA 115, 11567-11572 (2018).

18. Ramachandra Rao, S., Fliesler, S. J., Kotla, P., Nguyen, M. N. \& Pittler, S. J. Lack of overt retinal degeneration in a K42E dhdds knock-in mouse model of RP59. Cells 9, 896 (2020).

19. Ramachandra Rao, S. et al. Retinal degeneration caused by rod-specific dhdds ablation occurs without concomitant inhibition of protein $\mathrm{N}$-glycosylation. iScience 23, 101198 (2020).

20. Bar-El, M. L. et al. Structural characterization of full-length human dehydrodolichyl diphosphate synthase using an integrative computational and experimental approach. Biomolecules 9, 660 (2019).

21. Grabińska, K. A., Edani, B. H., Park, E. J., Kraehling, J. R. \& Sessa, W. C. A conserved C-terminal RXG motif in the NgBR subunit of cis-prenyltransferase is critical for prenyltransferase activity. J. Biol. Chem. 292, 17351-17361 (2017).

22. Ma, J. et al. Structural insights to heterodimeric cis-prenyltransferases through yeast dehydrodolichyl diphosphate synthase subunit Nus1. Biochem. Biophys. Res. Commun. 515, 621-626 (2019).

23. Edani, B. H. et al. Structural elucidation of the cis-prenyltransferase NgBR/ DHDDS complex reveals insights in regulation of protein glycosylation. Proc. Natl Acad. Sci. USA 117, 20794-20802 (2020).

24. Krissinel, E. \& Henrick, K. Inference of macromolecular assemblies from crystalline state. J. Mol. Biol. https://doi.org/10.1016/j.jmb.2007.05.022 (2007).

25. Ko, T. P. et al. Mechanism of product chain length determination and the role of a flexible loop in Escherichia coli undecaprenyl-pyrophosphate synthase catalysis. J. Biol. Chem. 276, 47474-47482 (2001).

26. Kharel, Y., Takahashi, S., Yamashita, S. \& Koyama, T. Manipulation of prenyl chain length determination mechanism of cis-prenyltransferases. FEBS J. 273, 647-657 (2006).

27. Tian, W., Chen, C., Lei, X., Zhao, J. \& Liang, J. CASTp 3.0: computed atlas of surface topography of proteins. Nucleic Acids Res. 46, W363-W367 (2018).

28. White, K. A. et al. Cancer-associated arginine-to-histidine mutations confer a gain in $\mathrm{pH}$ sensing to mutant proteins. Sci. Signal. https://doi.org/10.1126/ scisignal.aam9931 (2017).

29. Ho, B. K. \& Brasseur, R. The Ramachandran plots of glycine and pre-proline. BMC Struct. Biol. 5, 14 (2005).

30. Endo, S., Zhang, Y. W., Takahashi, S. \& Koyama, T. Identification of human dehydrodolichyl diphosphate synthase gene. Biochim. Biophys. Acta 1625, 291-295 (2003).

31. Wen, R., Lam, B. L. \& Guan, Z. Aberrant dolichol chain lengths as biomarkers for retinitis pigmentosa caused by impaired dolichol biosynthesis. J. Lipid Res. 54, 3516-3522 (2013).

32. Giladi, M. et al. Purification and characterization of human dehydrodolychil diphosphate synthase (DHDDS) overexpressed in E. coli. Protein Expr. Purif. 132, 138-142 (2017).

33. Edri, I. et al. Overexpression and purification of human cis-prenyltransferase in Escherichia coli. J. Vis. Exp. 126, e56430 (2017).

34. Kabsch, W. XDS. Acta Crystallogr. Sect. D Biol. Crystallogr. 66, 125-132 (2010).
35. Keegan, R. M. \& Winn, M. D. MrBUMP: an automated pipeline for molecular replacement. Acta Crystallogr. Sect. D Biol. Crystallogr. 64, 119-124 (2007).

36. Winn, M. D. et al. Overview of the CCP4 suite and current developments. Acta Crystallogr. Sect. D Biol. Crystallogr. 67, 235-242 (2011).

37. Adams, P. D. et al. PHENIX: a comprehensive Python-based system for macromolecular structure solution. Acta Crystallogr. Sect. D Biol. Crystallogr 66, 213-221 (2010).

38. Emsley, P. \& Cowtan, K. Coot: model-building tools for molecular graphics. Acta Crystallogr. Sect. D. Biol. Crystallogr. 60, 2126-2132 (2004).

39. Chen, V. B. et al. MolProbity: all-atom structure validation for macromolecular crystallography. Acta Crystallogr. Sect. D Biol. Crystallogr. https://doi.org/10.1107/S0907444909042073 (2010).

40. Yates, A. D. et al. Ensembl 2020. Nucleic Acids Res. https://doi.org/10.1093/ nar/gkz966 (2020).

41. Thompson, J. D., Gibson, T. J. \& Higgins, D. G. Multiple sequence alignment using ClustalW and ClustalX. Curr. Protoc. Bioinform. https://doi.org/ 10.1002/0471250953.bi0203s00 (2003).

42. Ben Chorin, A. et al. ConSurf-DB: An accessible repository for the evolutionary conservation patterns of the majority of PDB proteins. Protein Sci. https://doi.org/10.1002/pro.3779 (2020).

43. Abraham, M. J. et al. Gromacs: high performance molecular simulations through multi-level parallelism from laptops to supercomputers. SoftwareX 1-2, 19-25 (2015).

44. Kaminski, G. A., Friesner, R. A., Tirado-Rives, J. \& Jorgensen, W. L. Evaluation and reparametrization of the OPLS-AA force field for proteins via comparison with accurate quantum chemical calculations on peptides. J. Phys. Chem. B 105, 6474-6487 (2001).

45. Darden, T., York, D. \& Pedersen, L. Particle mesh Ewald: an N.log(N) method for Ewald sums in large systems. J. Chem. Phys. 98, 10089-10092 (1993).

46. Humphrey, W., Dalke, A. \& Schulten, K. VMD: visual molecular dynamics. J. Mol. Graph. https://doi.org/10.1016/0263-7855(96)00018-5 (1996).

\section{Acknowledgements}

We thank the staff of I03 at the Diamond Light Source for assistance with diffraction experimentation and Dr. Reuven Wiener for technical assistance with diffraction data collection. This work was performed in partial fulfillment of the requirements for a Ph.D degree of M.L.B.-E., Sackler Faculty of Medicine, Tel-Aviv University, Israel. This work was supported by the Israel Science Foundation (grant 1721/16) (Y.H.), the Israel Cancer Research Foundation grants 01214 (Y.H.) and 19202 (M.G.), and from the GermanIsraeli Foundation for Scientific Research and Development (grant No. I-2425-418.13/ 2016) (Y.H.). Support also came from the I-CORE Program of the Planning and Budgeting Committee and the Israel Science Foundation (grant 1775/12) (Y.H.), Recanati Foundation (M.G.), Marguerite Stolz Research Fellowship (Y.H.), Kahn foundation's Orion project, Tel Aviv Medical Center, Israel (M.G.), Israel Cancer Association (grant 20200037) (Y.H. and M.G.), and the Claire and Amedee Maratier Institute for the Study of Blindness and Visual Disorders, Sackler Faculty of Medicine, Tel-Aviv University (Y.H. and M.G.). Access to MS installation was funded by the EU Horizon 2020 grant EU_FT-ICR_MS project number 731077 and by CIISB LM2018127. P.M. and P.V. support from MEYS CZ funds CZ.1.05/1.1.00/02.0109 is gratefully acknowledged.

\section{Author contributions}

Conceptualization: Y.H. and M.G.; Methodology: Y.H. and M.G.; Investigation: M.L.B. E., P.V., A.Y., L.S., H.E., P.M., Y.H., and M.G.; Formal analysis: M.L.B.-E., A.Y., L.S., P.M., Y.H., and M.G.; Writing-original draft: Y.H. and M.G.; Writing-review and editing: M.L.B.-E., P.M., Y.H., and M.G.; Supervision: Y.H. and M.G.; Funding acquisition: Y.H. and M.G.

\section{Competing interests}

The authors declare no competing interests.

\section{Additional information}

Supplementary information is available for this paper at https://doi.org/10.1038/s41467 020-18970-z.

Correspondence and requests for materials should be addressed to Y.H. or M.G.

Peer review information Nature Communications thanks Sriganesh Ramachandra Rao and other, anonymous, reviewers for their contributions to the peer review of this work. Peer review reports are available.

Reprints and permission information is available at http://www.nature.com/reprints

Publisher's note Springer Nature remains neutral with regard to jurisdictional claims in published maps and institutional affiliations. 
(c) (i) Open Access This article is licensed under a Creative Commons Attribution 4.0 International License, which permits use, sharing, adaptation, distribution and reproduction in any medium or format, as long as you give appropriate credit to the original author(s) and the source, provide a link to the Creative Commons license, and indicate if changes were made. The images or other third party material in this article are included in the article's Creative Commons license, unless indicated otherwise in a credit line to the material. If material is not included in the article's Creative Commons license and your intended use is not permitted by statutory regulation or exceeds the permitted use, you will need to obtain permission directly from the copyright holder. To view a copy of this license, visit http://creativecommons.org/ licenses/by/4.0/.

(C) The Author(s) 2020 